\title{
Hardware-in-the-loop simulation system for space information networks
}

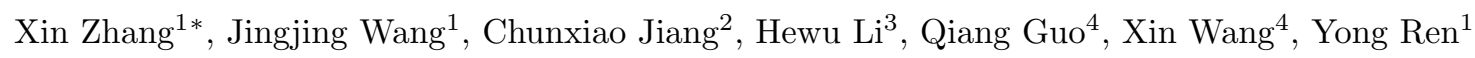 \\ 1. Department of Electronic Engineering, Tsinghua University, Beijing, 100084, China \\ 2. Tsinghua Space Center, Tsinghua University, Beijing, 100084, China \\ 3. Network Research Center, Tsinghua University, Beijing, 100084, China \\ 4. National Satellite Meteorological Center and the Key Laboratory of Radiometric Calibration \\ and Validation for Environmental Satellite, Meteorological Administration, Beijing, 100081, China \\ * Corresponding author, Email: zhangxin15@mails.tsinghua.edu.cn
}

\begin{abstract}
Thanks to the recent advances in SIS (Space Information Science) and increasingly mature technologies, network simulations have attracted much attention, which play vital roles in research on validation of the correctness of SIN's (Space Information Network) solutions. In this paper, we constitute an architecture of a hardware-in-the-loop simulation system, where we use a server to act as a real satellite of SINs. Moreover, we model the channel of the satellite network relying on real cables and servers by referring to the Corazza model. Furthermore, based on the data of real weather events, we propose a coordinate mode for reducing LEO's (Low Earth Orbit) communication delay and validate the efficiency of our proposed method on simulation platform by simplifying the problem to a shortest path problem.
\end{abstract}

Keywords: hardware-in-the-loop, network simulations, satellite channel model, architecture

\section{Introduction}

Owing to the indispensably strategic position and unique strength of various information accessing, multiple task coordination and power observation ability, the SIN has become the focus of national and international research and has been making rapid progress. The research on SIN has stretched into every corner such as resource allocation, protocol tuning, etc ${ }^{[1]}$. As the basis for each field, simulation is always an indispensable part in this area. For the simulation of space systems, costly hardware simulation makes it impossible for its unbearable time and money consumption. Over years, with the great progress of computer science, methods for simulation emerge. Due to the increasing complexity and the inter-relationship between the design of the processes and the design of the control system, computer-aided methods for modeling, simulation and also the design itself, are increasingly required. However, the pure software is limited to the model you choose. That is to say, the margin between your results and real values depends on how much your model is applicable to the problem. On the basis of the mathematical simulation, HIL (Hardware-In-the-Loop) simulation system is composed of the real parts, which are key

Manuscript received Sep. 25, 2017; accepted Nov. 10, 2017

This work is supported by the National Natural Science Foundation of China (No. 91338203). 
parts or links of the research and others realized by the simulation in the mathematical loops. The advantages of the HIL simulation system can be summarized as follows:

- It designs and validates the proposed architecture by controlling hardware and software without the necessity to establish a real process which costs much time and material.

- It runs without the need to operate real manipulations.

- It could offer reproducible and frequently repeatable experiments.

The HIL simulation system is characterized by the operation of real components in connection with realtime simulated components. Usually, the real system hardware used for series application has real time costs for experiments, which are usually too long to wait. In our system, we use a server and cables to simulate the satellite channel, which are easy to configure and to operate. The controlled process consists of software including Quagga, iPerf, STK (Satellite Tool Kit) and MATLAB, whose details are given in the following part.

In fact, HIL simulators are attracting more attention and there have been many demos completed for SIN. German Space Center developed an HIL simulation system called European Proximity Operations Simulator in 1999 to simulate satellite rendezvous operations ${ }^{[2]}$. There have been several examples of HIL simulators for simulating contact-dynamics operations of space systems, which have been applied to test ATV and HTV rendezvous sensors. Based on a 6-DOF Stewart platform, NASA/MSF developed an HIL simulator aiming at simulating the Space Shuttle berthing to the ISS (International Space Station $)^{[3]}$. The Canadian Space Agency also built the STVF (SPDM Task Verification Facility) using a giant 6-DOF customer-built hydraulic robot to simulate SPDM performing contact tasks on ISS ${ }^{[4]}$. US Naval Research Lab used two 6-DOF robotic arms to simulate satellite rendezvous for HIL testing rendezvous sensors ${ }^{[5]}$. Chinese scientists developed a dual-robot facility to simulate satellites on orbit servicing operations ${ }^{[6]}$. Benninghoff presented a method for closed loop rendezvous simulation with optical sensors so that the GNC (Guidance, Navigation and Control) system could cope with delayed measurements and enabled a stable approach ${ }^{[7]}$. In this paper, we introduce an HIL simulation system for SIN. Our contribution can be summarized as follows:

- We propose an architecture of an HIL simulation system in which we use a server to play the role of a real satellite for the simulation of a space information network.

- We provide the details of simulating a satellite channel relying on real cables and servers.

- With the help of the data of real weather events, we propose a coordinate mode for the LEO communication and validate the efficiency on the simulation platform.

The following parts are organized as follows. In section 2 , we provide a detailed introduction of the HIL simulation system including the architecture and interfaces. In section 3, we present the method of simulation of the satellite channel model. In section 4 and section 5, a simulation relying on the real weather events, i.e. Typhoon Haiyan is operated on our HIL simulation system, which demonstrates the application of research on SIN, followed by our conclusions and future work in section 6 .

\section{The HIL simulation system}

In this section, we introduce our HIL simulation system from three aspects, i.e. architecture, interfaces, and implementations.

\subsection{Architecture}

The core of the HIL simulation system consists of servers and PCs. The core is the basis for upper level manipulations including protocol tuning, designing resource allocation algorithms, making realtime demo, etc., as shown in Fig. 1. The PC plays the role of a control center, which distributes the commands including the topology of network and control messages. STK and MTALB accomplish the 
pre-computation jobs. Quagga's mission is to configure the network and the link according to the data generated by the PC, while iPerf reflects the performance of SIN.

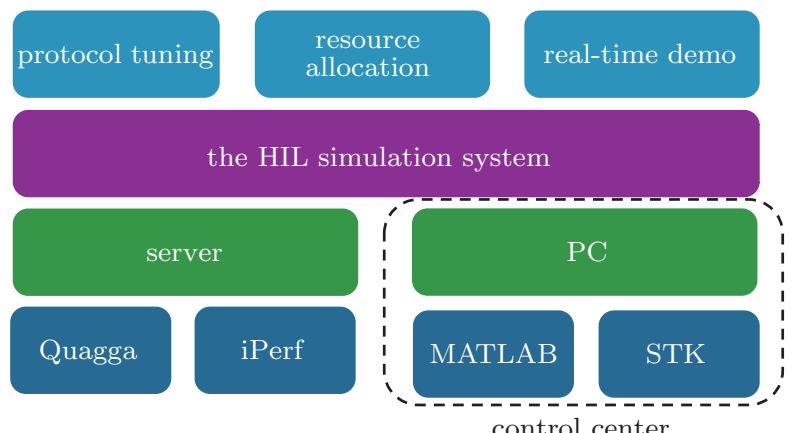

Figure 1 The architecture of the HIL simulation system

\section{$2.2 \quad$ Interfaces}

STK is a software with powerful functions for satellite research and simulation, especially for its visualization, the most exciting of which is the interface to connect with MATLAB, with which we can manipulate STK in MATLAB command line modes or scripts.

The connector, i.e. Mexconnect, can link MATLAB with STK via a TCP process in a full duplex transmission mode, with which we can not only control the STK by MATLAB but also can fetch the intermediate data from STK to make further research. By using Mexconnect tools, we can create various STK objects such as creating a new scene both in a 2D form and a 3D form, setting a satellite, a ground station; we can configure the flight parameters of aircrafts, establish the connected relations among different nodes, generate the analysis report about attitude, coverage area, visibility, physical parameters such as distance, velocity, etc., which can be restored or manipulated by MATLAB. The PC can interact with servers through the Linux command line or shell scripts. All of these interfaces are shown in Fig. 2.
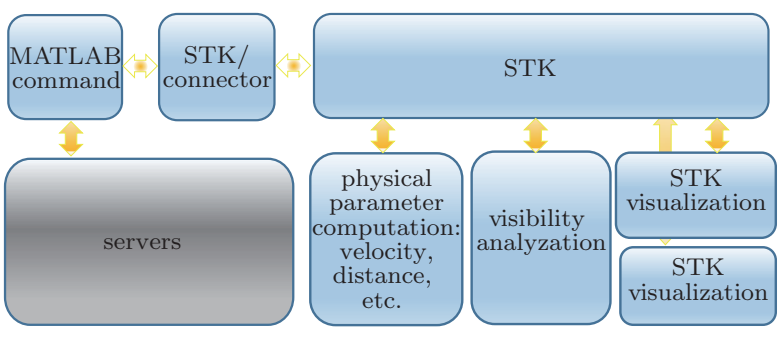

Figure 2 Interfaces of the HIL simulation system

\subsection{Implementation}

Our platform is composed of 9 Dell servers with CentOS 7.0 operating system, which is shown in Fig. 3, on which we configure the open-source software, i.e. Quagga, which supports OSPFv3 and ISIS in various beta states. IS-IS for IPv4 is believed to be usable. Moreover, OSPFv3 and IS-IS for IPv6 have known issues. Each server represents a node, which can be a LEO satellite, GEO satellite or a ground station. As a result, our simulation platform can also be used for protocol tuning in both IP layer and transport layer such as TCP tuning, etc.

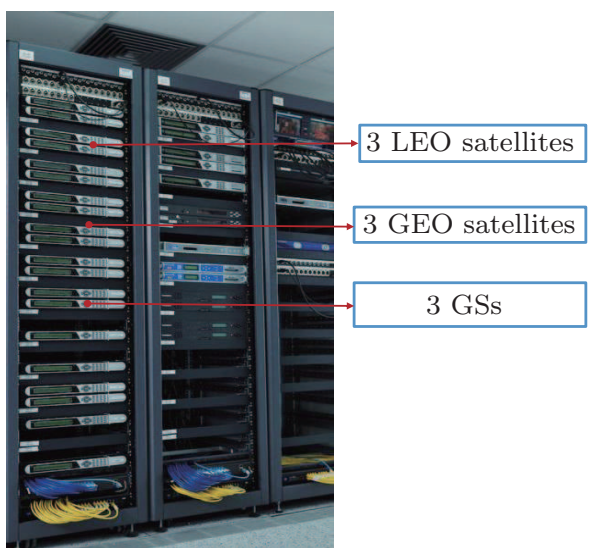

Figure 3 Physical equipment

To reflect the real-time performance of the satellite links, we utilize iPerf to report quality of service. iPerf is an open source software for active measurements of the maximum achievable bandwidth on IP networks. It supports tuning of various parameters related to timing, buffers and protocols (TCP, UDP, SCTP with IPv4 and IPv6). For each test, it reports the bandwidth, loss, and other parameters. Both 


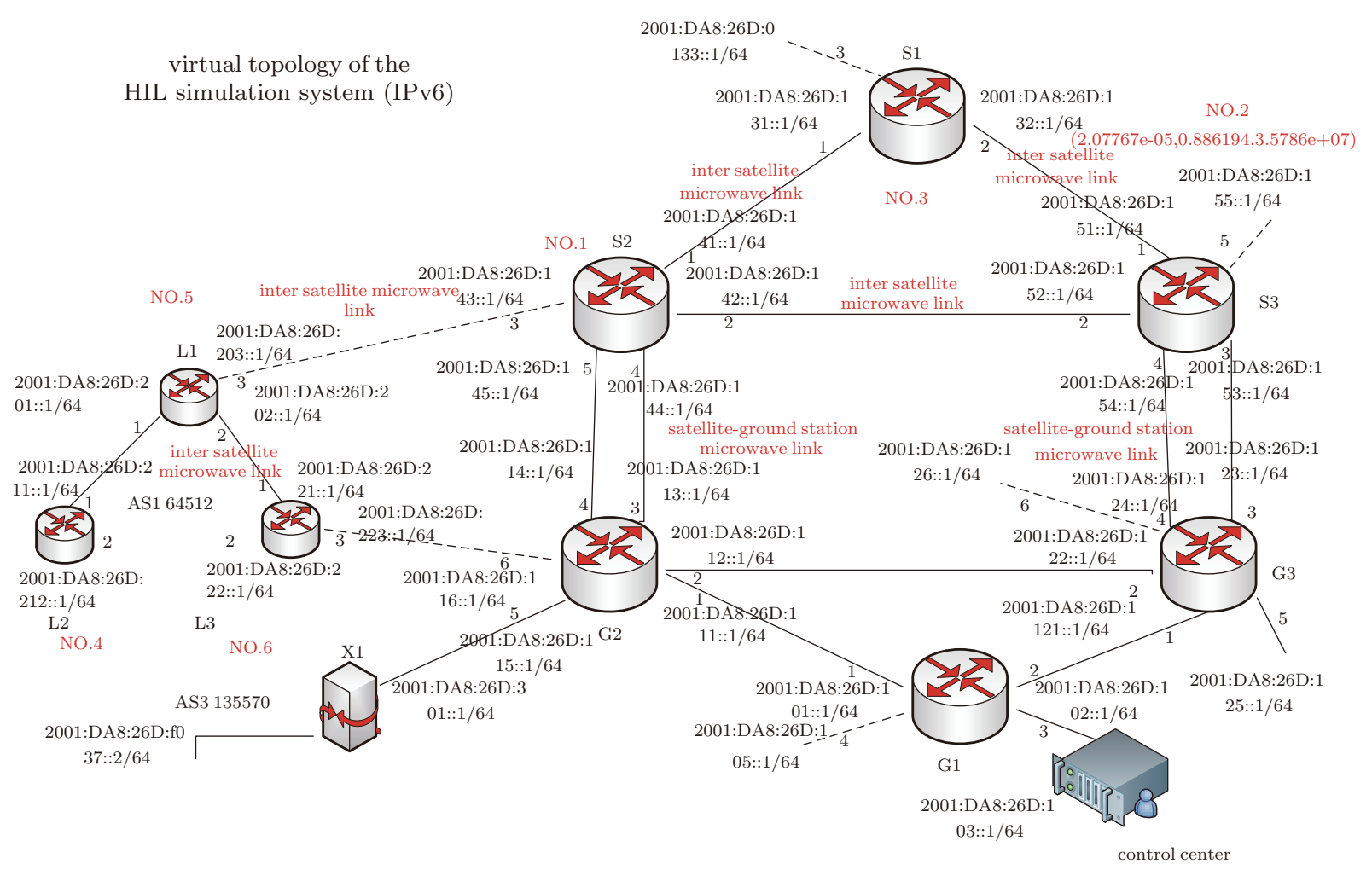

Figure 4 Virtual topology of the HIL simulation system

tools run on each server and Quagga configures the topology which is produced by STK-MATLAB and sends data through iPerf.

There are 3 GEO satellites, 3 LEO satellites and 3 GSs (Ground Stations) as shown in Tab. 1. The virtual topology is shown in Fig. 4, where each server is configured with an IPv6 address and the control center can distribute the topology of each snapshot and the time table of the generated data, which also includes data size.

Table 1 Components of system

\begin{tabular}{ccc}
\hline satellites & number & nodes in Fig. 3 \\
\hline LEO & 3 & $L_{1}, L_{2}, L_{3}$ \\
GEO & 3 & $S_{1}, S_{2}, S_{3}$ \\
GS & 3 & $G_{1}, G_{2}, G_{3}$ \\
\hline
\end{tabular}

\section{Satellite channel models}

In this section, we use the data rate, delay and error rates, which can be computed by the following mode, to simulate the satellite channel. On our platform, we realize 3 typical channel models, which are the C. Loo model, the Corazza model and the Lutz model, where the propagation characteristics of the $\mathrm{L}$ band satellite mobile communication channel are modeled according to the masking of the signal in the propagation path. Furthermore, the C. Loo model is only applicable in rural channel, while the Corazza model and Lutz model can be used to depict many kinds of channel including highway, village, uptown and city.

\subsection{Inter-satellite link}

The ISL (Inter-Satellite Link) can be depicted by the following equations. EIRP (Equivalent Isotropically Radiated Power) can be calculated as:

$$
E I R P=P_{T} * G_{T},
$$

where $P_{T}$ is the transmitting power and $G_{T}$ is the transmitting antenna gain. Free space loss can be 
calculated as:

$$
L_{f}=\left(\frac{4 \pi R}{\lambda}\right)^{2}=\left(\frac{4 \pi R f}{3 \times 10^{8}}\right)^{2},
$$

where $R$ is the distance between transmitter, $\lambda$ is the carrier wavelength of the receiver and $f$ is transmitting frequency.

For different bands, we have:

$$
\begin{cases}K a: & \frac{G}{T} \geqslant 40+20 \log \left(\frac{f}{25}\right) \\ X: & \frac{G}{T} \geqslant 35+20 \log \left(\frac{f}{7.9}\right) \\ S: & \frac{G}{T} \geqslant 21+20 \log \left(\frac{f}{2.2}\right)\end{cases}
$$

where $G / T$ is the quality factor.

According to the link equation, we can acquire the SNR of the channel and other arguments are shown in Tab. 2. The signal-to-interference ratio can be given by:

$$
\frac{E_{\mathrm{b}}}{N_{0}}=E I R P-L_{f}+\frac{G}{T}+228.6-10 \lg R_{b},
$$

where $R_{b}$ is the data rate of the link.

Table 2 Arguments for channel simulation

\begin{tabular}{ccccc}
\hline Link & $G_{t} / \mathrm{dB}$ & $G_{T} / \mathrm{dB}$ & $R_{b} / \mathrm{bit}^{-\mathrm{s}^{-1}}$ & $f / \mathrm{GHz}$ \\
\hline LEO-LEO & 8.1 & 33.05 & $9.3 \mathrm{M}$ & 23.28 \\
GEO-LEO & 8.1 & 33.05 & $9.3 \mathrm{M}$ & 2 \\
LEO-GS & 28.1 & 33.05 & $25 \mathrm{M}$ & 23.28 \\
\hline
\end{tabular}

\subsection{Satellite-ground station}

Although the rainfall in some low frequency bands, such as the C-band, does not cause much attenuation, they are too crowded. For higher band, the factors which hinder satellite links will increase with the frequency, especially for rain attenuation. If the frequency is higher than $10 \mathrm{GHz}$, the rain attenuation is able to decrease the signal level, even resulting in aggravating the quality of the entire satellite link. Specially, during vital meteorological disasters, the influence is definitely not negligible. Therefore, the rain attenuation must be taken into consideration when using the Ku-band or the Ka-band. The
Ka-band communication channel is a slow flat fading channel. Therefore, the channel multiplicative fading is a constant during a reasonable $T$ to infer the following equation:

$$
r(t)=\alpha \mathrm{e}^{\mathrm{j} \phi} s(t),
$$

where $r(t)$ and $s(t)$ are the received signal and sending signal, respectively. $\alpha$ and $\phi$, whose probability distributions are relevant to the envelope and the phase of the equivalent channel, obey normal distribution as shown as follows.

$$
p(\alpha)=\frac{1}{\sqrt{2 \pi} \sigma_{1}} \mathrm{e}^{-\frac{\left(\alpha-\mu_{1}\right)^{2}}{2 \sigma_{1}^{2}}},
$$

as well as

$$
p(\phi)=\frac{1}{\sqrt{2 \pi} \sigma_{2}} \mathrm{e}^{-\frac{\left(\phi-\mu_{2}\right)^{2}}{2 \sigma_{2}^{2}}},
$$

where $p(\alpha)$ represents the probability density function of the envelop and $p(\phi)$ is the probability density function of the phase, whose means are $\mu_{1}, \mu_{2}$ and variances are $\sigma_{1}, \sigma_{2}$, respectively. Tab. 3 presents the parameters in some typical weathers ${ }^{[8]}$.

Considering both rain attenuation and environment, the link equation can be formulated as ${ }^{[9]}$ :

$$
\begin{aligned}
\frac{E_{\mathrm{b}}}{N_{0}}= & E I R P-L_{f}+\frac{G_{R}}{T_{S}}+228.6 \\
& -10 \lg R+L_{r}+L_{c},
\end{aligned}
$$

where $L_{r}$ represents the part caused by rain attenuation and $L_{c}$ represents the part caused by environment.

Table 3 Means and variances

\begin{tabular}{cccccc}
\hline \multirow{2}{*}{ weather } & \multicolumn{2}{c}{$\begin{array}{c}\text { amplitude statistic } \\
\text { parameter }\end{array}$} & & \multicolumn{2}{c}{$\begin{array}{c}\text { phase statistic } \\
\text { parameter }\end{array}$} \\
\cline { 2 - 3 } \cline { 5 - 6 } mean & variance & & mean & variance \\
\hline fine & 0.455 & 0.00056 & & 0.0079 & 0.00381 \\
split & 0.662 & 0.02 & & -0.0089 & 0.03077 \\
moderate rain & 0.483 & 0.00003 & & 0.0088 & 0.00546 \\
rainstorm & 0.436 & 0.0138 & & 0.0068 & 0.00414 \\
\hline
\end{tabular}

The Corazza model takes the received signal as the product of a log-normal distribution and a Rice 
distribution. Concretely, the conditional probability distribution of the envelope of received signal is subject to:

$$
\begin{aligned}
p_{r}(r \mid S)= & \frac{2(K+1) r}{S^{2}} \mathrm{e}^{-\frac{(K+1) r^{2}}{S^{2}}} \\
& \times I_{0}\left[\frac{2 r}{S} \sqrt{K(K+1)}\right],
\end{aligned}
$$

where $K$ is the ratio of the power of direct signal and multi-path signal, and $S$ is shadow fading, which is subject to the log-normal distribution:

$$
p_{S}(S)=\frac{1}{S \sqrt{2 \pi} \sigma_{S}} \exp \left[-\frac{(\ln S-\mu)^{2}}{2 \sigma_{S}^{2}}\right] .
$$

As we all know, for a ground station, the elevation of satellite is time-variant, which incurs the changing propagation environment. These changes are embodied in the changing arguments which are $K, \mu$ and $\sigma$. Using polynomial regression and the experiential arguments in the Tab. 4, we can depict the channel fully. Finally we use a weighting sine model to simulate the Rice distribution ${ }^{[10]}$.

$$
\begin{gathered}
K(\alpha)=K_{0}+K_{2} \alpha^{2}+K_{3} \alpha^{3}, \\
\mu(\alpha)=\mu_{0}+\mu_{2} \alpha^{2}+\mu_{3} \alpha^{3},
\end{gathered}
$$

and

$$
\sigma(\alpha)=\sigma_{0}+\sigma_{2} \alpha^{2}+\sigma_{3} \alpha^{3} .
$$

Table 4 Fitting coefficient

\begin{tabular}{cccc}
\hline fitting coef. & village & suburbs & city \\
\hline$K_{0}$ & 2.731 & 1.75 & -13.6 \\
$K_{1}$ & -0.1074 & 0.067 & 0.965 \\
$K_{2}$ & $2.774 \times 10^{-3}$ & 0 & -0.01663 \\
$K_{3}$ & 0 & 0 & $1.187 \times 10^{-4}$ \\
$\mu_{0}$ & -20.25 & -52.12 & -1.998 \\
$\mu_{1}$ & 0.9919 & 2.758 & $-9.919 \times 10^{-4}$ \\
$\mu_{2}$ & -0.0168 & -0.048 & 0.00152 \\
$\mu_{3}$ & $9.502 \times 10^{-5}$ & $2.714 \times 10^{-4}$ & $-1.266 \times 10^{-5}$ \\
$\sigma_{S_{0}}$ & 4.5 & 7.8 & 8 \\
$\sigma_{S_{1}}$ & -0.05 & -0.3542 & -0.3741 \\
$\sigma_{S_{2}}$ & 0 & $6.5 \times 10^{-3}$ & $6.125 \times 10^{-3}$ \\
$\sigma_{S_{3}}$ & 0 & $-3.958 \times 10^{-5}$ & $-3.333 \times 10^{-5}$ \\
\hline
\end{tabular}

\section{Simulations}

The climate observation mission is divided into 2 parts, one of which is generated by GEO, while the other is generated by LEO. The data from GEO are generated every one hour with constant coverage area and they can communicate with the GS, as shown in Fig. 5. Since LEO is dynamic relative to the earth, it is not always able to communicate with GS, which results in many hours of delay for the data for analysis until it is visible for GS.

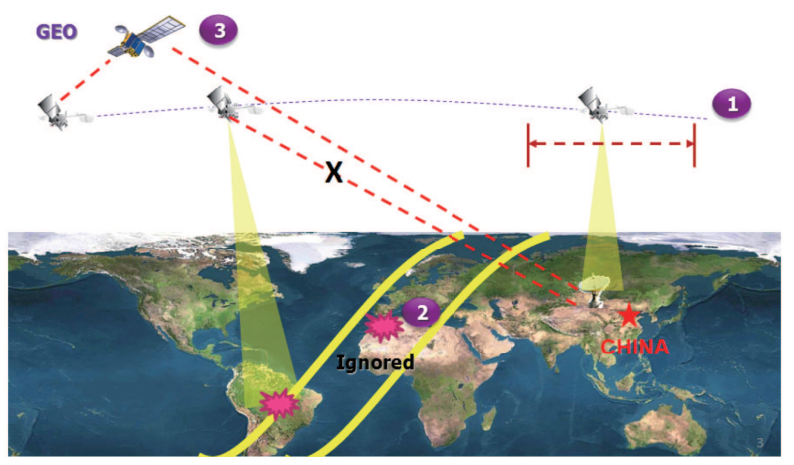

Figure 5 Non-cooperative observation

To address the problem above and overcome the limitation of the non-cooperative mode, we need to propose some cooperative modes and validate on the HIL system. However, up till now, there is no ISL between weather satellites, where our initiative comes from. However, the most fundamental step to fulfill the cooperative observation is the static links between satellites. With the advantages of lowattitude-orbits, little communication delay, high resolution for observation and easiness for networking, the LEO satellites are highly respected to improve the performance of space-based information network, including earth observing satellites. Here, we suppose there exists links between LEO satellites.

Suppose there are two usable ground stations: Beijing (40N,116E), Guangzhou (23N,113E). The orbit data can be found on the Satellite Catalog ${ }^{[11]}$. Their orbit parameters are shown in Tab. 5 . 
Table 5 Orbit parameters

\begin{tabular}{|c|c|c|c|c|c|}
\hline satellite & inclination $/^{\circ}$ & right ascension of ascending node $/^{\circ}$ & argument of perigee $/{ }^{\circ}$ & eccentricity & semi-major axis $/ \mathrm{m}$ \\
\hline FY-2D & 4.0417 & 65.4559 & 313.4402 & 0.0001272 & 42151000 \\
\hline FY-2E & 1.7654 & 64.8015 & 211.9959 & 0.0005102 & 42151000 \\
\hline FY-2F & 1.2663 & 77.1573 & 225.5153 & 0.0002440 & 42151000 \\
\hline FY-3C & 98.6775 & 21.2518 & 73.2245 & 0.0000513 & 7207000 \\
\hline NOAA & 99.1960 & 323.2507 & 336.6291 & 0.0013911 & 7225000 \\
\hline
\end{tabular}

\subsection{Data set}

As the most authoritative organization, NSMC (National Satellite Meteorological Centre $)^{[12]}$ acquires every batch of data collected by Chinese satellites such as FY series, or rented satellites such as NOAA (USA), MTSAT (USA), etc. The data are combined with their theory and experiences in analyzing weather events to make decisions and further improve the accuracy of weather prediction.

Taking typhoon as an example, by current technology for analysis, sensors on satellites used to analyze the typhoon include visible and infrared spin scan radiometer (VISSR, integrated in FY-2D, FY-2E, FY-2F), Microwave Temperature Sounder (MWTS, integrated in FY-3C) and Advanced Microwave Sounding Unit (AMSU-A AMSU-B, integrated in NOAA), whose data generation intervals are given in Tab. 6. And the data used for analyzing are filtered by meteorologists, as shown in Tab. 7 .

Table 6 Sensors

\begin{tabular}{ccc}
\hline satellite & data type & generation interval/min \\
\hline FY-2D & VISSR & 60 \\
FY-2E & VISSR & 60 \\
FY-2F & VISSR & 60 \\
FY-3C & MWTS & 15 \\
NOAA & AMSU-A AMSUB & 15 \\
\hline
\end{tabular}

\subsection{Topology}

Since the topology of space network is timecontinuous and dynamic, we use the discrete time slots to discretize the continuous time, just like the snapshots which are taken as constant time intervals.
Each interval is so short that the topology can be regarded as unchanged. By means of this, we acquire a series of static topology. On the basis of the satellite channel model, we can compute quality of each link in the network according to the real satellite arguments including data rates, delays and error rates, which is introduced in the following parts.

\subsection{Channel}

We use the model elaborated in section 3 to generate the channel parameter of each snapshot.

\subsection{Optimization}

We first distinguish the non-coordinate mode and the coordinate mode. The non-coordinate mode is the traditional mode without the ISLs, while the coordinate mode is a mode in which the two LEO satellites could communicate with each other, which means one can be the relay of the other. Viewing all the ground stations as a sink node, a satellite visible to any ground station is equivalent to its ability to transport data to the ground. Here we give the definition of distance of each data batch.

$$
\begin{aligned}
& D_{i j}(t+1)= \\
& \left\{\begin{array}{rr}
\min \left(\left|t_{i, j, \text { next }}-t\right|, N-\left|t_{i, j, \text { next }}-t\right|\right), \\
D_{i j}(t)=0, \\
D_{i j}(t)-1, & D_{i j}(t)>0,
\end{array}\right.
\end{aligned}
$$

where $t_{i, j \text {,next }}$ indicates the index of the slot when node $i$ and node $j$ are visible to each other, while $N$ is the least common multiple of all the satellites' periods, which is also the number of time slots. 
Table 7 Data form

\begin{tabular}{ccccc}
\hline satellite & data type & generation interval/min & rise time & data size (M) \\
\hline FY-2D & VISSR & 60 & Year.Month.Day.Hour.Second & $\mathrm{xx}$ \\
\hline
\end{tabular}

\subsection{Computing the fastest route}

Therefore, starting from the definition of distance and targeting at the minimum distance, which is equivalent to the minimum delay, the problem is converted into a SPP (Shortest Path Problem) with a dynamic but certain cost for each snapshot. Here, we adopt the classical Floyd algorithm ${ }^{[13]}$ to compute the route of each batch by renewing the distance matrix $\boldsymbol{D}$ and by creating the path matrix $\boldsymbol{P}$ to print the path, which is shown in Algorithm 1 and Algorithm 2. A remarkable fact is that the path output by Algorithm 2 is a reversed sequence. To acquire the normal sequence, it is helpful to set up a stack.

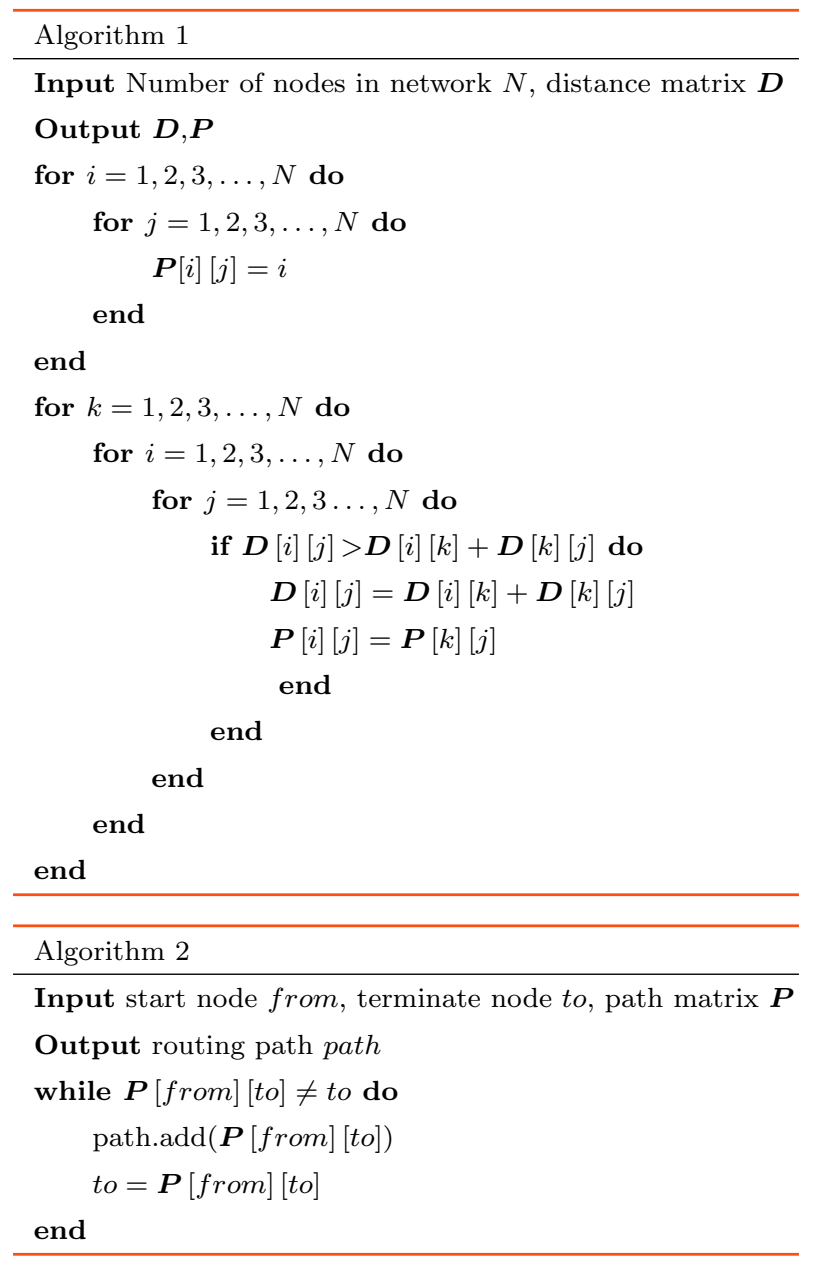

\section{Experimental evaluation}

In this section, we present the results of our simulations. Fig. 6 and Fig. 7 are the visualizations of our simulation scene and the topology generates when the programme is running. As shown in Fig. 8 for FY-3C, the delay of each data batch is plotted on the $Y$-axis. The $X$-axis indicates the index of each batch. The delay in non-coordinate mode is painted in blue while the delay in coordinate mode is painted in green.

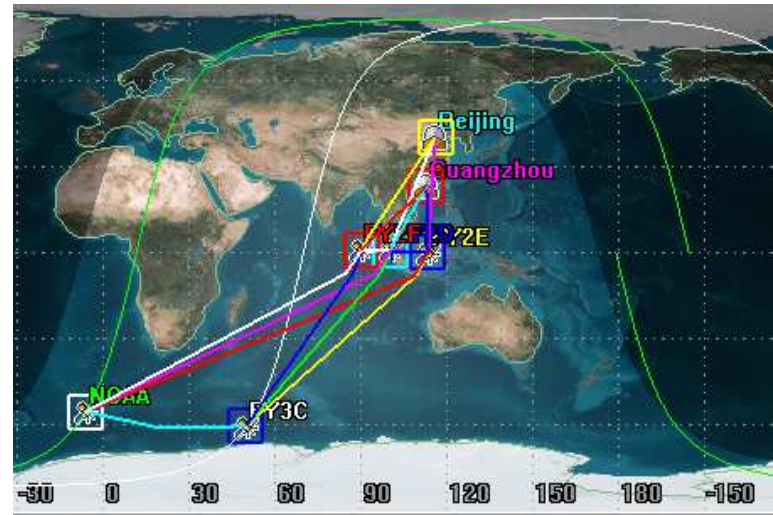

Figure 6 2D-visualization

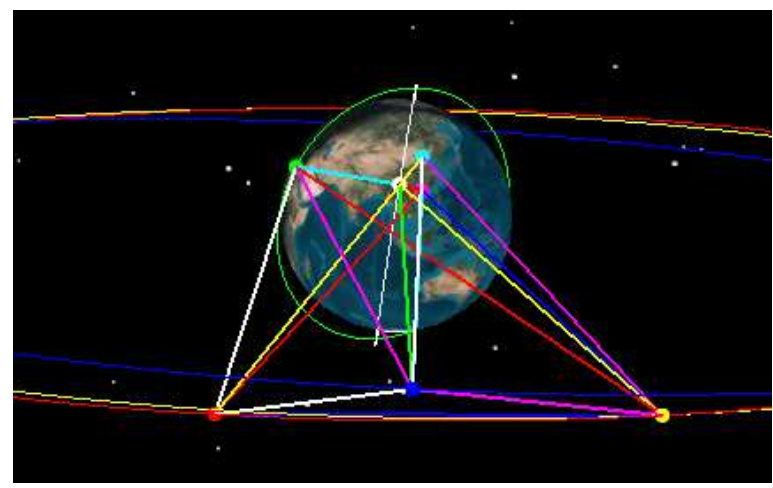

Figure 7 3D-visualization

The most obvious character is that the delay in the coordinate mode is much less than that in noncoordinate mode. The same result is revealed in Fig. 9. Comparing the means of delays in two modes, 


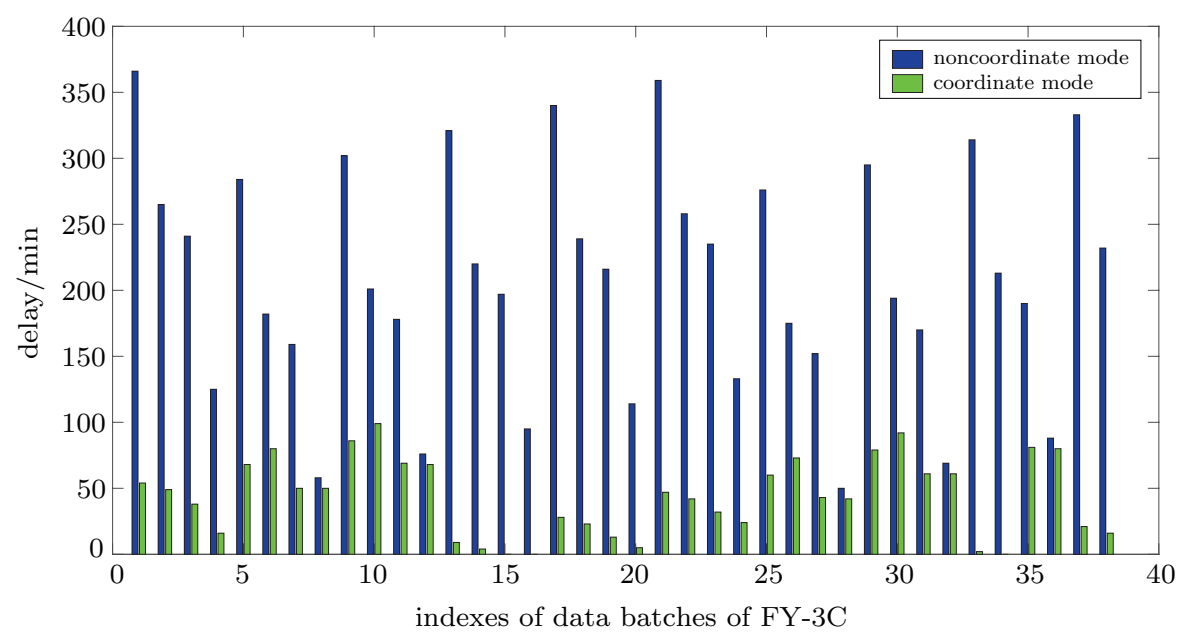

Figure 8 Delays of the data batches of FY-3C

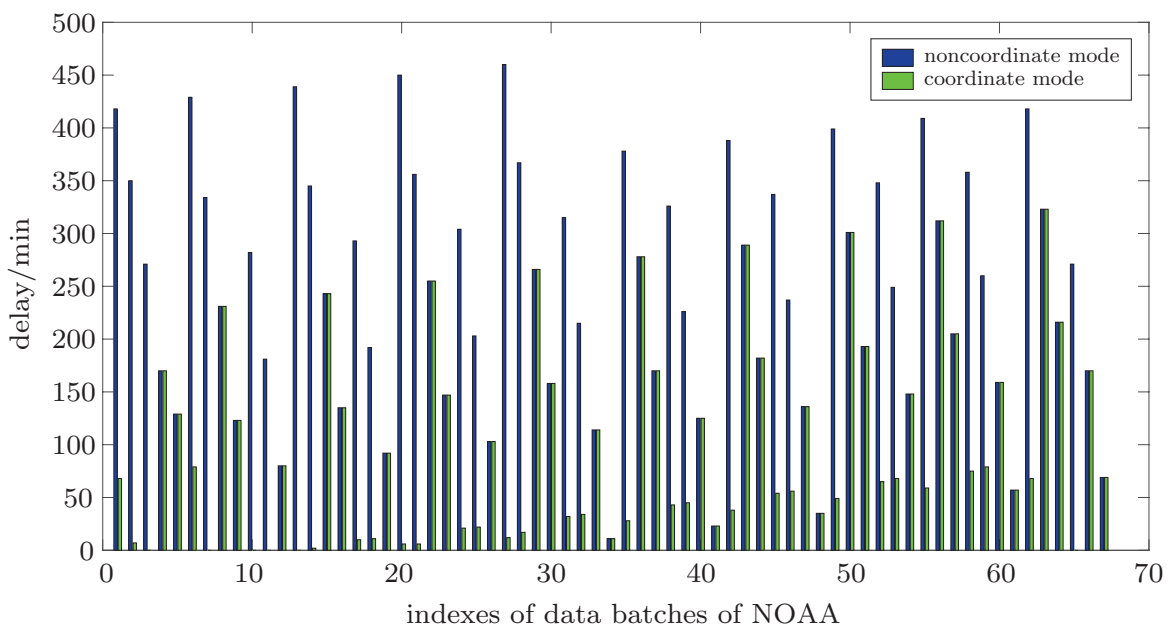

Figure 9 Delays of the data batches of NOAA

we notice that coordinate mode can reduce delays by $67.46 \%$ for FY-3C and $68.85 \%$ for NOAA, which strongly confirms the validity of the coordination mode and informs us of the necessity to step further towards ISLs and the urge to realize the network of satellites.

\section{Conclusions and future work}

In this paper, we give a realization of an HIL simulation system for SIN using STK and MATLAB as the satellite links. STK is expert in attitude analysis, orbit analysis, establishing its priority in design constellations, performing validations and making demos. MATLAB provides powerful computa- tion, straightforward data visualization and general restoration, which can be used by other software. Replacing satellites with servers and the expensive real satellite links with cables not only lowers the costs, but also increases the flexibility of configuration and manipulation, most importantly, the ability to research higher layer problems in space information networks. The HIL system is able to reflect the statistic character including delay and error rates of real links, which makes it more powerful than a software simulation. Moreover, low cost and less time consumption are of benefit to the system, which provides a valuable reference for research on SINs and we give various experiential models under different weathers. Finally, by reproducing the Ty- 
phoon Haiyan and giving the definition of distance, we compare the delay in a coordination mode with that in a traditional mode to prove the efficiency of the former. In addition, the system can be regarded as a paradigm for further intelligent programming such as mission programming, resource allocation, etc., including but not confined to protocol layer. For these reasons, the HIL system will be a powerful tool for future research on SIN. However, the current efforts are only the first step and it is far from taking full advantage of the platform.

\section{References}

[1] J. J. Wang, C. X. Jiang, H. J. Zhang, et al. Aggressive congestion control mechanism for space systems [J]. IEEE aerospace and electronic systems magazine, 2017, 31(3): 28-33.

[2] R. Krenn, B. Schaefer. Limitations of hardware-in-theloop simulations of space robotics dynamics using industrial robots [C]//European Space Agency, ESA SP-440, 1999: 681-686.

[3] S. Ananthakrishnan, R. Teders, K. Alder. Role of estimation in real-time contact dynamics enhancement of space station engineering facility [J]. IEEE robotics and automation magazine, 1996, 3(3): 20-28.

[4] J. C. Piedboeuf, J. D. Carufel, F. Aghili, et al. Task verification facility for the Canadian special purpose dextrous manipulator [C]//IEEE International Conference on Robotics and Automation, Detroit, 1999, 3: 10771083.

[5] R. Bell, T. Morphopoulos, J. Pollack, et al. Hardwarein-the-loop tests of an autonomous gn\&c system for onorbit servicing [C]//AIAA-LA Section/SSTC Responsive Space Conference, 2003.

[6] W. F. Xu, B. Liang, Y. S. Xu, et al. A ground experiment system of free-floating space robot for capturing space target $[\mathrm{J}]$. Journal of intelligent and robotic systems, 2007, 48(2): 187-208.

[7] H. Benninghoff, F. Rems, T. Boge. Development and hardware-in-the-loop test of a guidance, navigation and control system for on-orbit servicing $[\mathrm{J}]$. Acta astronautica, 2014, 102(102): 67-80.

[8] E. Matricciani. Transformation of rain attenuation statistics from fixed to mobile satellite communication systems [J]. IEEE transactions on vehicular technology, 1995, 44(3): 565-569.

[9] Z. Wang. Research on channel characteristics and modeling of high-speed mobile communication links [D]. Beijing: Beijing Institute of Technology, 2015.
[10] M. Ptzold. Mobile radio channels. Second edition [M]. UK: WILEY, 2012.

[11] Satellite catalog [EB/OL]. http://celestrak.com/satcat/ search.asp.

[12] National Satellite Meteorological Centre [EB/OL]. http://www.nsmc.org.cn/NSMC/Home/Index.html.

[13] R. W. Floyd. Algorithm 97: shortest path [J]. Communications of the ACM, 1962, 5(6): 345.

\section{About the authors}

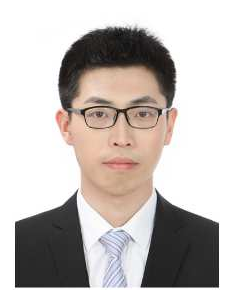

Xin Zhang [corresponding author] received his B.E. degree in information engineering from Beijing University of Posts and Telecommunications. He is now pursuing his master's degree at Department of Electronic Engineering, Tsinghua University. His research interests include space information network and optimization. (Email: zhangxin15@mails.tsinghua.edu.cn)

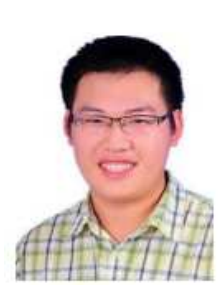

Jingjing Wang (S'14) received his B.S. degree (Hons.) in electronic information engineering from the Dalian University of Technology in 2014. He is currently pursuing his Ph.D. degree with the Complex Engineered Systems Lab, Tsinghua University, Beijing, China. His research interests include the complex networkbased modeling and the game theory in wireless communication and networking. (Email: chinaeephd@gmail.com)

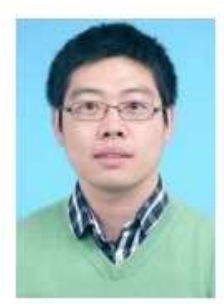

Chunxiao Jiang (S'09-M'13-SM'15) received his B.S. degree (Hons.) in information engineering from Beihang University in 2008, and his Ph.D. degree (Hons.) from Tsinghua University, Beijing, China, in 2013. From 2011 to 2012, he was with the Signals and Information Group, Department of Electrical and Computer Engineering, University of Maryland. From 2013 to 2016, he was a Post-Doctoral Researcher with the Department of Electronic Engineering, Tsinghua University. He is currently an assistant research fellow with the Tsinghua Space Center, Tsinghua University. His research interests include the applications of game theory and queuing theory in wireless communication and networking. He received the Best Paper Award from the IEEE GLOBECOM in 2013, Tsinghua Outstanding Distinguished Doctoral Dissertation in 2013, the Best Student Paper Award from the IEEE Global Conference on Signal and Information Processing in 2015, the Tsinghua Outstanding Postdoc Award in 2015, the Beijing Distinguished Graduated Student Award, and the Chinese National Fellowship. (Email: jchx@tsinghua.edu.cn) 


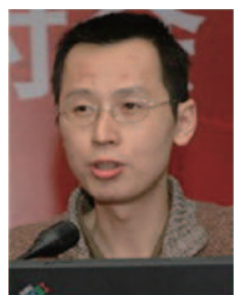

Hewu Li received his M.S. and Ph.D. degrees in Computer Science from Tsinghua University. His thesis focused on the performance optimization of Broadband Wireless Internet. He is now an assistant professor of School of Information Science and Technology, Tsinghua University, and Director of Wireless and Mobile Network research laboratory, Network Research Center. His research interests include mobile wireless network architecture, broadband wireless access technology, mobility architecture in next generation network, P2P SIP, and wireless network performance optimization. He led or participated more than 5 government funded research projects and has experience in leading international collaboration research projects, such as Nortel research funding and China-Japan joint project on IPv6. He has published over 15 journal and conference papers. (Email: lihewu@cernet.edu.cn)

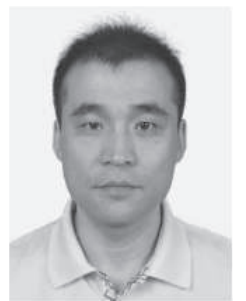

Qiang Guo received his M.S. degree in signal and information processing and Ph.D. degree in electronic science and technology, where his work focused on specifications, including spatial resolution and detection sensitivity, and onorbit evaluation for spaceborne remotely sensed instrument, from the Shanghai Institute of Technical Physics, Chinese Academy of Sciences, Shanghai, China, in 2001 and 2003, respectively. From 2003 to 2005 , he worked with a postdoctoral position to carry out some kernel data preprocessing, i.e., geolocation for the nextgeneration geostationary meteorological satellite in China, stray-light on-ground analysis for on-orbit satellites, and remotely sensed imagery quality evaluation, for native meteorological satellite in the Chinese Academy of Meteorological Sciences. Since 2005, he has been an associate professor and then a professor with the National Satellite Meteorological Center, China Meteorological Administration, Beijing, China. He is currently the deputy designer general of the ground-segment systems for both Fengyun-2 and Fengyun- 4 serial satellites. His research interests include on-orbit radiometric calibration, geolocation, and specification evaluation for spaceborne instruments. (Email: guoqiang@cma.gov.cn)

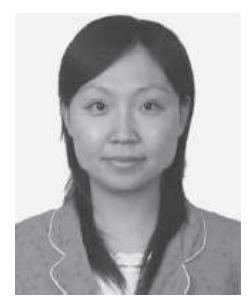

Xin Wang received her Ph.D. degree from the School of Physics, Peking University, Beijing, China, in 2008. During the period of doctoral study, her main research interests were tropical cyclones and tropical climatology by using multiple satellite data, and the atmospheric radiation characteristics on convective cloud based on model and satellite data. Since 2008, she has been a senior engineer with the National Satellite Meteorological Center, China Meteorological Administration, Beijing. She is now working in the leader duty of typhoon satellite monitoring and a deputy director designer of the application demonstration subsystem in the ground-segment systems for Fengyun-4 serial satellites. Her research interests include tropical cyclones and their internal mesoscale strong convective interaction mechanism, and geostationary satellite calibration verification and improvement in typhoons and convective weather. (Email: xinwang@cma.gov.cn)

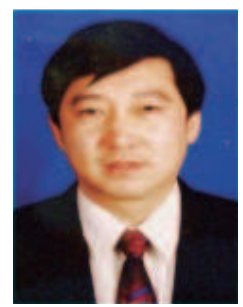

Yong Ren (M'11-SM'16) received his B.S., M.S. and Ph.D. degrees from the Harbin Institute of Technology, China, in 1984, 1987, and 1994, respectively, all in electronic engineering. He held a postdoctoral position with the Department of Electronics Engineering, Tsinghua University, China, from 1995 to 1997 . He is currently a professor with the Department of Electronics Engineering and the director of the Complexity Engineered Systems Lab, Tsinghua University. He holds 12 patents and has authored or co-authored over 100 technical papers on the behavior of computer network, $\mathrm{P} 2 \mathrm{P}$ network, and cognitive networks. He has served as a reviewer of IEICE transactions on communications, Digital signal processing, Chinese physics letters, Chinese journal of electronics, Chinese journal of computer science and technology, and Chinese journal of aeronautics. His current research interests include complex systems theory and its applications to the optimization and information sharing of the Internet, Internet of Things and ubiquitous network, cognitive networks, and cyber-physical systems. (Email: reny@tsinghua.edu.cn) 